Research Article

\title{
Theoretical Study of Chloro-N-(4-methoxybenzylidene)aniline Derivatives as Corrosion Inhibitors for Zinc in Hydrochloric Acid
}

\author{
S. Kumar, ${ }^{1}$ D. G. Ladha, ${ }^{1}$ P. C. Jha, ${ }^{2}$ and N. K. Shah ${ }^{1}$ \\ ${ }^{1}$ Department of Chemistry, School of Sciences, Gujarat University, Ahmedabad 380009, Gujarat, India \\ ${ }^{2}$ School of Chemical Sciences, Central University of Gujarat, Sector 30, Gandhinagar 382030, Gujarat, India \\ Correspondence should be addressed to N. K. Shah; sureshthakur80@yahoo.com
}

Received 28 July 2013; Accepted 9 November 2013

Academic Editor: Yanqing Lai

Copyright (c) 2013 S. Kumar et al. This is an open access article distributed under the Creative Commons Attribution License, which permits unrestricted use, distribution, and reproduction in any medium, provided the original work is properly cited.

\begin{abstract}
The density functional theory (DFT) was used to investigate the corrosion inhibition of three inhibitors on zinc. Quantum chemical parameters such as the energy of highest occupied molecular orbital $\left(E_{\mathrm{HOMO}}\right)$, the energy of lowest unoccupied molecular orbital $\left(E_{\mathrm{LUMO}}\right)$, energy gap $(\Delta E)$, hardness $(\eta)$, softness $(\sigma)$, electrophilicity index $(\omega)$, the fraction of electrons transferred $(\Delta N)$ from inhibitor molecule to the metal surface, energy change when both processes occur, namely, charge transfer to the molecule and backdonation from the molecule $\left(\Delta E_{\text {backdonation }}\right)$, natural charge $\left(q_{N}\right)$, and Fukui functions have been calculated by using B3LYP/6$31+G(d)$ basis set. The relation between the inhibition efficiency and quantum chemical parameters has been discussed in order to elucidate the inhibition mechanism of the chloro-N-(4-methoxybenzylidene)aniline derivatives.
\end{abstract}

\section{Introduction}

Quantitative structure activity relationship (QSAR) has been a subject of intense interest in many disciplines of chemistry. In addition, the results of quantum chemical calculations are obtained without laboratory measurements, thus saving time and equipment and alleviating safety and disposal concerns $[1,2]$. Density functional theory (DFT) has a potential application towards the design and development of organic corrosion inhibitors in corrosion field [3]. These facts have made quantum calculations to be very powerful tool for studying corrosion inhibition mechanism. Zinc is one of the most vital nonferrous metals having extensive use in metallic coating [4]. The use of zinc sheets as roofing materials and in galvanization of steel makes the study of its corrosion an important theme [5]. Zinc is often attacked by aggressive media, such as acids, bases, and salt solutions [6-8].

In acidic solutions, zinc has high solubility and dissolves with the formation of $\mathrm{Zn}^{2+}$ ions. Since the solubility of zinc decreases with increasing $\mathrm{pH}$ in acidic solutions, precipitation of $\mathrm{Zn}(\mathrm{OH})_{2}$ occurs when a certain $\mathrm{pH}$ value is reached. In alkaline solutions, with $\mathrm{pH}>9$, the solubility of zinc increases with increasing $\mathrm{pH}$, and in the high $\mathrm{pH}$ range, zinc oxide and hydroxides tend to dissolve with the formation of zincate ions. Zinc oxide is a common corrosion product in diluted solutions of salts such as $\mathrm{NaCl}$ or $\mathrm{Na}_{2} \mathrm{SO}_{4}$ with zinc hydroxide present in various amounts as a minor component. For scale removal and cleaning of zinc surface with acid solutions, the use of organic inhibitors is one of the most practical methods for protection against corrosion, especially in acidic media [9-13]. The aim of this work is to study the effect of quantum chemical parameters of 2Chloro-N-(4-methoxybenzylidene)aniline (2-CNMBA), 3chloro-N-(4-methoxybenzylidene)aniline (3-CNMBA), and 4-chloro-N-(4-methoxybenzylidene)aniline (4-CNMBA) on the inhibition efficiency of corrosion of zinc by using density functional theory (DFT) methods. For this purpose, the relation between the inhibition efficiencies of these compounds with quantum parameters has been investigated. The Fukui functions and local softness(s) values obtained were utilised to elucidate the reactive sites in the studied molecules. 


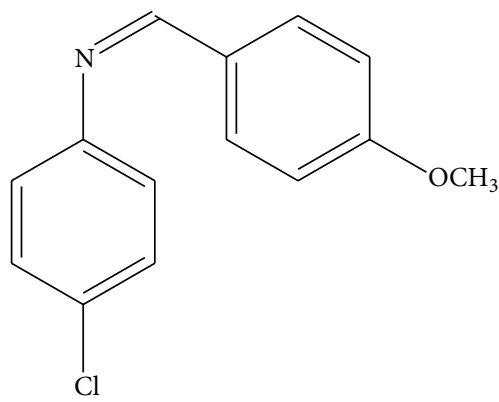

(a) 4-CNMBA

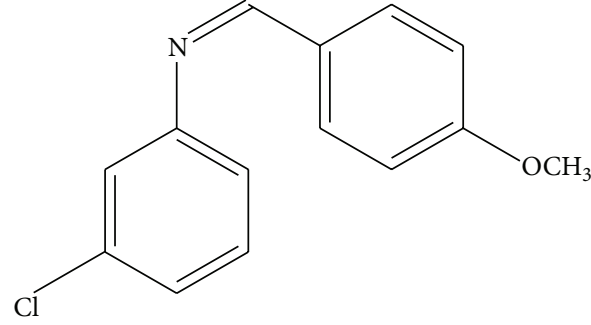

(b) 3-CNMBA

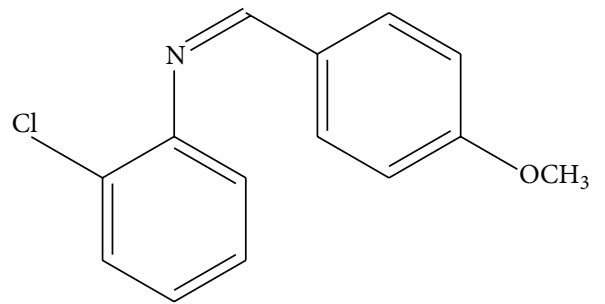

(c) 2-CNMBA

FIGURE 1: Molecular structure of studied compounds (a) 4-CNMBA, (b) 3-CNMBA, and (c) 2-CNMBA.

\section{Materials and Methods}

2.1. Preparation of Schiff Bases. The Schiff bases were synthesized by condensation of 4-methoxybenzaldehyde with 2chloroaniline, 3-chloroaniline, and 4-chloroaniline in the presence of ethyl alcohol as per the procedure described by Shah et al. [14]. 2-CNMBA (boiling point: $362.4^{\circ} \mathrm{C}$, registry number is not available) is dark reddish liquid, 3-CNMBA (boiling point: $335.9^{\circ} \mathrm{C}$, registry number: $\left.24776-57-6\right)$ is brownish liquid, and 4-CNMBA (melting point: $335.9^{\circ} \mathrm{C}$, registry number: 15485-22-0) is a white crystalline solid. These compounds are insoluble in water but soluble in ethanol, methanol, ethyl acetate, and THF. These compounds have previously been tested as inhibitors for Al-Mg alloy in $\mathrm{HCl}$ [15]. The molecular structures of the Schiff bases are shown in Figures 1(a), 1(b), and 1(c). The compounds were characterised through IR spectroscopy as shown in Figures 2(a), 2(b), and 2(c). 4-CNMBA has $-\mathrm{C}-\mathrm{H}$ stretching of aromatic ring $\left(3131 \mathrm{~cm}^{-1}\right),>\mathrm{C}=\mathrm{N}-\left(1648 \mathrm{~cm}^{-1}\right),-\mathrm{C}-\mathrm{O}-\mathrm{C}-$ stretching in aromatic ring $\left(1257 \mathrm{~cm}^{-1}\right),-\mathrm{C}-\mathrm{Cl}$ stretching in aromatic ring $\left(766 \mathrm{~cm}^{-1}\right)$ and $3-\mathrm{CNMBA}$ has $-\mathrm{C}-\mathrm{H}$ stretching of aromatic ring $\left(3164 \mathrm{~cm}^{-1}\right),>\mathrm{C}=\mathrm{N}-\left(1634 \mathrm{~cm}^{-1}\right),-\mathrm{C}-\mathrm{O}-\mathrm{C}-$ stretching in aromatic ring $\left(1240 \mathrm{~cm}^{-1}\right)$ and $-\mathrm{C}-\mathrm{Cl}$ stretching in aromatic ring $\left(737 \mathrm{~cm}^{-1}\right) .2-\mathrm{CNMBA}$ has $-\mathrm{C}-\mathrm{H}$ stretching aromatic ring $\left(3120 \mathrm{~cm}^{-1}\right),>\mathrm{C}=\mathrm{N}\left(1642 \mathrm{~cm}^{-1}\right),-\mathrm{C}-\mathrm{O}-\mathrm{C}-$ stretching in aromatic ring $\left(1263 \mathrm{~cm}^{-1}\right)$ and $-\mathrm{C}-\mathrm{Cl}$ stretching in aromatic ring $\left(744 \mathrm{~cm}^{-1}\right)$.

2.2. Weight Loss Measurements. Rectangular specimens of electrolytic zinc of size $3.0 \mathrm{~cm} \times 3.0 \mathrm{~cm}$ (thickness $=0.139 \mathrm{~cm})$ with a small hole of about $2 \mathrm{~mm}$ diameter just near one end of the specimen were used for the determination of the corrosion rate. The specimens were grinded using successively " 0 " to "0000" Oakey emery paper. The polishing was done using jewellers rouge, which gave mirror-like finish. The specimens were washed with ethanol and finally with distilled water and then dried at room temperature. The dried and weighed specimens were exposed to $230 \mathrm{~mL}$ of $0.5 \mathrm{M} \mathrm{HCl}$ solution containing controlled additions of various Schiff bases in the range of $0.0-0.5 \%(\mathrm{w} / \mathrm{v})$. One specimen was suspended by a pyrex glass hook in each beaker of the test solution, which was open to the air at $35 \pm 0.5^{\circ} \mathrm{C}$, to the same depth of about $1.5 \mathrm{~cm}$ below the surface of the liquid. The weight of the specimen before and after immersion was determined using mettler balance-M5 type.

The percentage inhibition efficiencies $\left(\delta_{w} \%\right)$ were calculated using the following equation:

$$
\text { Inhibitor efficiency }\left(\delta_{w} \%\right)=\frac{W_{u}-W_{i}}{W_{u}} \times 100 \text {, }
$$

where $W_{u}$ is weight loss of zinc in uninhibited acid and $W_{i}$ is weight loss of zinc in inhibited acid.

2.3. Quantum Chemical Calculations. All the quantum chemical calculations have been carried out with Gaussian 09 programme package [16]. In our calculation we have used B3LYP, a hybrid functional of the DFT method, which consists of the Becke's three parameters; exact exchange functional B3 combined with the nonlocal gradient corrected correlation functional of Lee-Yang-Par (LYP) has been used along with 6$31+\mathrm{G}(\mathrm{d})$ basis set. In the process of geometry optimisation for the fully relaxed method, convergence of all the calculations has been confirmed by the absence of imaginary frequencies. The aim of our calculation is to calculate the following quantum chemical indices: the energy of highest occupied molecular orbital $\left(E_{\mathrm{HOMO}}\right)$, the energy of lowest unoccupied molecular orbital $\left(E_{\mathrm{LUMO}}\right)$, energy gap $(\Delta E)$, hardness $(\eta)$, softness $(\sigma)$, electrophilicity index $(\omega)$, the fraction of electrons transferred $(\Delta N)$ from inhibitor molecule to the metal surface, and energy change when both processes occur, namely, charge transfer to the molecule and backdonation from the molecule $\left(\Delta E_{\text {backdonation }}\right)$, and correlate these with the experimental observations.

The number of electrons transferred $(\Delta N)$ from the inhibitor molecule to the metal surface was calculated according to Pearson $[17,18]$ as shown in (2)

$$
\Delta N=\frac{\chi_{\mathrm{Zn}}-\chi_{\mathrm{inh}}}{2\left(\eta_{\mathrm{Zn}}+\eta_{\mathrm{inh}}\right)} .
$$

Here $\chi_{\mathrm{Zn}}$ and $\chi_{\mathrm{inh}}$ denote the absolute electronegativity of zinc and the inhibitor molecules, respectively. $\eta_{\mathrm{Zn}}$ and $\eta_{\text {inh }}$ denote the absolute hardness of zinc and the inhibitor molecules, respectively. The fraction of electron transferred $(\Delta N)$ is 


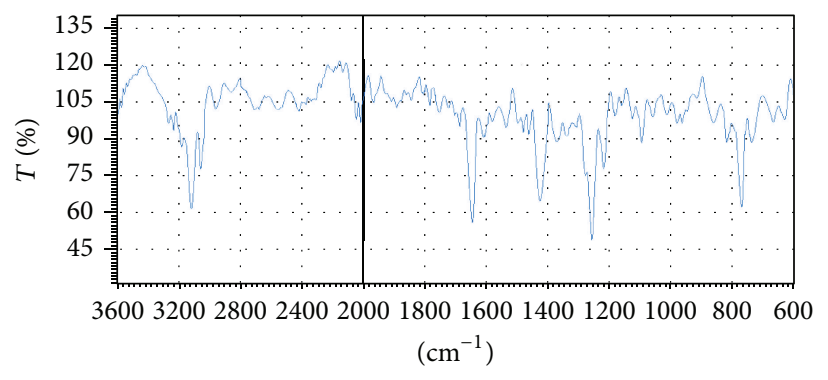

(a) 4-CNMBA

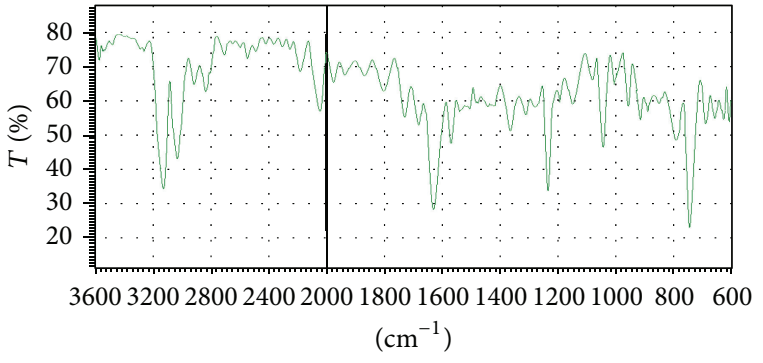

(b) 3-CNMBA

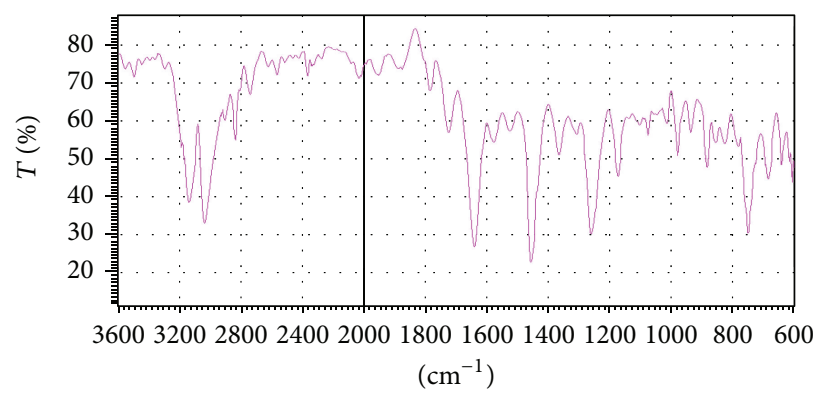

(c) 2-CNMBA

FIGURE 2: IR spectra of studied Schiff bases (a) 4-CNMBA, (b) 3-CNMBA, and (c) 2-CNMBA.

calculated for zinc metal, and the experimental work function of polycrystalline zinc $(8.126 \mathrm{eV})[19,20]$ was employed for electronegativity, and global hardness of zinc was taken as zero since $I \approx A$ for bulk zinc. Ionisation potential $(I)$ and electron affinity $(A)$ are related to $E_{\mathrm{HOMO}}$ and $E_{\mathrm{LUMO}}$ as follows [21, 22]:

$$
\begin{gathered}
I=-E_{\text {HOMO }}, \\
A=-E_{\text {LUMO }} .
\end{gathered}
$$

The quantities absolute electronegativity $(\chi)$ and global hardness $(\eta)$ are related to electron affinity $(A)$ and ionization energy $(I)$ as follows [23]:

$$
\begin{array}{ll}
\chi=\frac{I+A}{2}, & \chi=\frac{E_{\mathrm{HOMO}}+E_{\mathrm{LUMO}}}{2}, \\
\eta=\frac{I-A}{2}, & \eta=\frac{E_{\mathrm{HOMO}}+E_{\mathrm{LUMO}}}{2} .
\end{array}
$$

Global softness is defined as [24]

$$
\sigma=\frac{1}{\eta}
$$

Recently, a new global chemical reactivity parameter has been introduced and known as electrophilicity index $(\omega)$. It is defined as $[19,25]$

$$
\omega=\frac{\chi^{2}}{2 \eta}
$$

This is proposed as a measure of the electrophilic power of a molecule. When two molecules react, which one will act as an electrophile or nucleophile will depend on the fact that which one has a higher or lower electrophilicity index. The electrophilicity is a measure of reactivity that quantitatively classifies the global electrophilic nature of a molecule within a reactive scale. Partial atomic charges and atomic Fukui functions are good indicators of selectivity, that is, the region on the molecule on which certain type of reactions is likely to occur. The regions of a molecule where the Fukui function is large are chemically softer than the regions where the Fukui function is small. By involving the hard soft acid base (HSAB) principle in the local sense, one may establish the behaviour of the different sites with respect to hard or soft reagents [26]. The Fukui functions have been calculated by taking the finite difference approximations from natural population analysis of atoms in all the Schiff bases:

$$
\begin{array}{ll}
f^{+}=\left(q_{N+1}-q_{N}\right) & \text { for electrophilic attack, } \\
f^{-}=\left(q_{N}-q_{N-1}\right) & \text { for nucleophilic attack, } \\
f^{o}=\left(\frac{q_{N+1}+q_{N-1}}{2}\right) \text { for free radical attack. }
\end{array}
$$

Here " $q$ " is natural charge of atom in the molecule, that is, the electron density at a point " $r$ " in space around the molecule. The " $N$ " corresponds to the number of electrons in the neutral molecule. " $N+1$ " corresponds to an anion, with an electron added to the LUMO of the neutral molecule. " $N$ 1 " corresponds to a cation, with an electron removed from the $\mathrm{HOMO}$ of the neutral molecule. All the calculations were performed at the ground state geometry.

Reactivity of similar atoms of different molecules can also be compared by calculating condensed softness indices 
from the Fukui functions and global softness $(\sigma)$ by using the following expression [27]:

$$
\begin{gathered}
s^{+}=\left(f^{+}\right) \cdot \sigma, \\
s^{-}=\left(f^{-}\right) \cdot \sigma, \\
\Delta E_{\text {backdonation }}=-\frac{\eta}{4} .
\end{gathered}
$$

According to the simple charge transfer model for donation and backdonation of charges, proposed by Gomez et al. [28, 29], if both processes occur (namely, charge transfer to the molecule and backdonation from the molecule) the energy change is directly proportional to the hardness $(\eta)$ of the molecule as shown above in (10).

\section{Results and Discussions}

3.1. Weight Loss Measurements. The effect of addition of different chloro-N-(4-methoxybenzylidene)aniline derivatives at various concentrations on the zinc corrosion in $0.5 \mathrm{M}$ $\mathrm{HCl}$ solutions is studied by weight loss measurements at $35^{\circ} \mathrm{C} \pm 0.5^{\circ} \mathrm{C}$ after $30 \mathrm{~min}$ immersion. The values of inhibition efficiencies (\%IE) obtained from (1) and corrosion rates obtained from weight loss measurements for the three chloro-N-(4-methoxybenzylidene)aniline derivatives at different concentrations in $0.5 \mathrm{M} \mathrm{HCl}$ are listed in Table 1 . It is found that inhibition efficiency increases with increasing inhibitor concentration, while corrosion rate decreases with inhibitor concentration. The inhibition of zinc corrosion can be attributed to the adsorption of the inhibitors at the zinc/ $\mathrm{HCl}$ solution interface. All Schiff bases are good inhibitors showing more than $99 \%$ inhibition efficiency at $0.5 \%(\mathrm{w} / \mathrm{v})$ concentrations. Good performance of these compounds as corrosion inhibitors for zinc in $0.5 \mathrm{M} \mathrm{HCl}$ solutions may be due to the presence of the $>\mathrm{C}=\mathrm{N}, \mathrm{OCH}_{3}$ groups and benzene rings in their structures. From Table 1, it is clear that the order of the inhibition efficiency of these Schiff bases is as follows: 4 -CNMBA $\geq 3$-CNMBA $>2$-CNMBA.

3.2. Computational Study. We have used DFT method in order to understand if any structural differences, induced by the position of chlorine on the N-(4-methoxybenzylidene) aniline, are related to the observed differences of the corrosion inhibition efficiency. DFT calculations and correlations were performed on the three inhibitors, that is, 2-CNMBA, 3-CNMBA, and 4-CNMBA. It is generally accepted that the values of $E_{\mathrm{HOMO}}$ indicate the electron donating ability of the molecule and the inhibition efficiency increases with increasing $E_{\mathrm{HOMO}}$ value. High $E_{\mathrm{HOMO}}$ values indicate that the molecule has a tendency to donate electrons to the unoccupied orbitals of the metal with low energy empty orbital [30]. An increase in $E_{\mathrm{HOMO}}$ values facilitates the adsorption and therefore corrosion inhibition. $E_{\mathrm{LUMO}}$ indicates the ability of the molecules to accept electrons; with lower $E_{\mathrm{LUMO}}$ values, a greater adsorption ability and better corrosion inhibition can be expected [31]. The optimized structures of all three Schiff bases, namely, (a) 4-CNMBA, (b) 3-CNMBA, and (c)
TABLE 1: Corrosion parameters for zinc in $0.5 \mathrm{M} \mathrm{HCl}$ in the absence and presence of various concentrations of Schiff bases at $35 \pm 0.5^{\circ} \mathrm{C}$.

\begin{tabular}{lccc}
\hline Inhibitor & $\begin{array}{c}\text { Concentration } \\
(\mathrm{M})\end{array}$ & $\begin{array}{c}\text { Corrosion rate } \\
\left(\mathrm{mg} / \mathrm{dm}^{2}\right)\end{array}$ & $\delta_{w} \%$ \\
\hline \multirow{4}{*}{ 4-CNMBA } & Blank & 7930 & \\
& $6.1 \times 10^{-4}$ & 1041 & 86.87 \\
& $1.1 \times 10^{-4}$ & 66 & 99.17 \\
& $1.6 \times 10^{-3}$ & 23 & 99.71 \\
& $2.0 \times 10^{-3}$ & 20 & 99.74 \\
& $6.1 \times 10^{-4}$ & 1 & 99.98 \\
\hline \multirow{3}{*}{ 3-CNMBA } & $8.1 \times 10^{-4}$ & 1168 & 85.27 \\
& $1.2 \times 10^{-3}$ & 76 & 99.04 \\
& $1.6 \times 10^{-3}$ & 25 & 99.68 \\
& $2.0 \times 10^{-3}$ & 20 & 99.74 \\
& $6.1 \times 10^{-4}$ & 6 & 99.92 \\
\hline \multirow{3}{*}{-CNMBA } & $8.1 \times 10^{-4}$ & 4267 & 46.19 \\
& $1.2 \times 10^{-3}$ & 2682 & 66.18 \\
& $1.6 \times 10^{-3}$ & 589 & 92.57 \\
& $2.0 \times 10^{-3}$ & 229 & 97.12 \\
& & 71 & 99.1 \\
\hline
\end{tabular}

2-CNMBA with numbering schemes are depicted in Figure 3 which is shown below.

The total energy values given in Table 2 show the stability of the molecules which is 4 -CNMBA $>3$-CNMBA $\geq 2$ CNMBA. This trend is the same as that experimentally.

The value of $E_{\mathrm{HOMO}}$ of 4-CNMBA is the highest which correlates with the experimentally determined inhibition efficiency and the values of $E_{\text {LUMO }}$ favour 3-CNMBA. The separation energy, $\Delta E=\left(E_{\mathrm{LUMO}}-E_{\mathrm{HOMO}}\right)$, is an important parameter and it is a function of reactivity of the inhibitor molecule towards the adsorption on metallic surface. As $\Delta E$ decreases, the reactivity of the molecule increases leading to increase in the inhibition efficiency of the molecule [31]. The calculation from Table 2 shows the decreasing trend for the $\triangle E$ : 2-CNMBA $(4.2179 \mathrm{eV})>3$-CNMBA $(4.1906 \mathrm{eV})$ $>4$-CNMBA $(4.1362 \mathrm{eV})$ which follows the same order of inhibition efficiency as obtained experimentally.

The calculations show an obvious correlation between the molecular area of the molecules and the inhibition efficiency. The inhibition efficiency increases as molecular area increases due to the increase of the contact area between molecule and surface. It is clear from Table 2 that 4 -CNMBA has the highest molecular area that probably increases its adsorption on the metal surface and hence increases the inhibition efficiency. Global hardness $(\eta)$ and global softness $(\sigma)$ are important properties to measure the molecular stability and reactivity. A hard molecule has a large energy gap and a soft molecule has a small energy gap. Soft molecules are more reactive than hard ones because they could easily offer electrons to an acceptor. In the corrosion system, the inhibitor acts as a Lewis base, while the metal acts as a Lewis acid. Bulk metals are soft acids and thus soft base inhibitors are most effective for acidic corrosion inhibition of these metals. It is also in accordance 


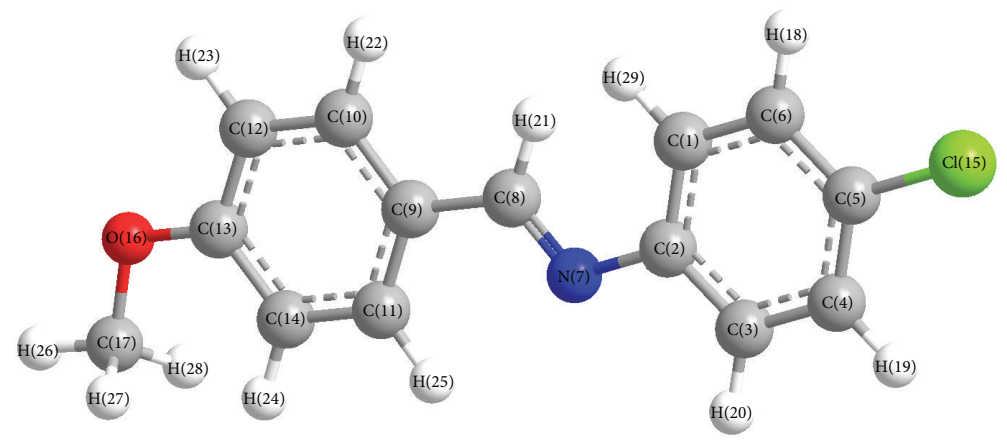

(a) 4-CNMBA

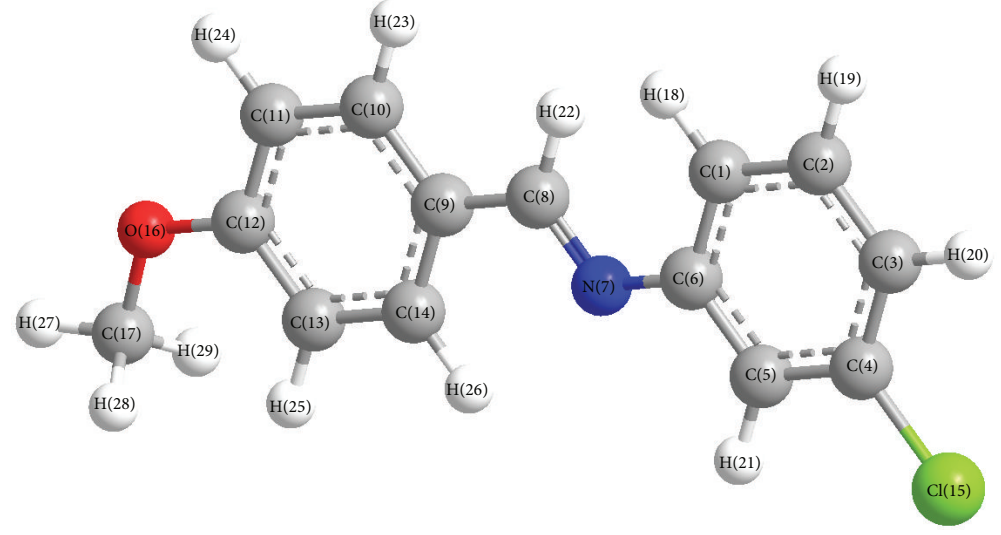

(b) 3-CNMBA

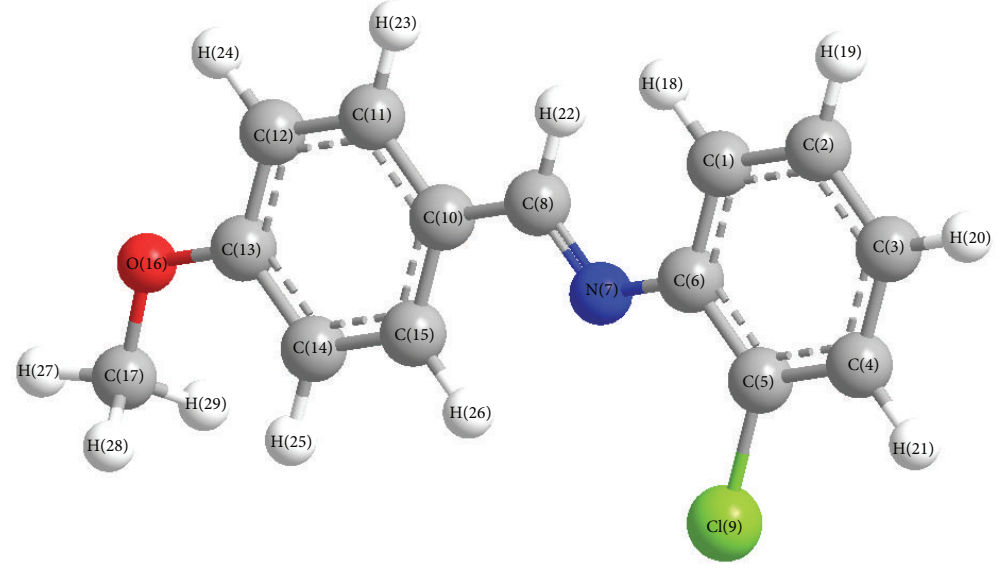

(c) 2 -CNMBA

Figure 3: Optimized structures of the Schiff bases (a) 4-CNMBA, (b) 3-CNMBA, and (c) 2-CNMBA.

with HSAB principle. Normally, the molecule with least value of global hardness (hence highest value of global softness) is expected to have the highest inhibition efficiency [27]. It is clear from the calculation that 4 -CNMBA has the lowest hardness and the highest softness which follow the same trend as obtained experimentally.

The electrophilicity index $(\omega)$, which shows the ability of the inhibitor molecule to accept electrons, follows the trend: 3-CNMBA (3.766) > 4-CNMBA (3.7123) > 2 CNMBA (3.6152). 3-CNMBA exhibits the highest value of electrophilicity (Table 2) which confirms its highest capacity to accept electrons. There is strong correlation between this property and inhibition efficiency.

The number of electrons transferred $(\Delta N)$ was also calculated and tabulated in Table 2 . The $\Delta N<3.6$ indicates the tendency of a molecule to donate electrons to the metal surface; therefore, the inhibition efficiency increases with increasing electron donating ability of these inhibitors to the metal surface [29] and it follows the order 3-CNMBA $(0.991) \leq 2$-CNMBA $(1.008)<4$-CNMBA (1.0172). The results indicate that $\triangle N$ value of $4-C N M B A$ is highest which correlates strongly with experimental inhibition efficiencies. Thus, 
TABLE 2: The calculated quantum chemical parameters for studied inhibitors obtained with the DFT at the B3LYP/6-31+G(d) method.

\begin{tabular}{|c|c|c|c|c|}
\hline S. N. & Quantum chemical properties & 4-CNMBA & 3-CNMBA & 2-CNMBA \\
\hline 1 & Total energy $(\mathrm{au})$ & -2941.06 & -2941.05 & -2940.98 \\
\hline 2 & $E_{\text {Номо }}(\mathrm{eV})$ & -5.9866 & -6.0683 & -6.0139 \\
\hline 3 & $E_{\text {LUMO }}(\mathrm{eV})$ & -1.8504 & -1.8776 & -1.7960 \\
\hline 4 & $\Delta E(\mathrm{eV})$ & 4.1362 & 4.1906 & 4.2179 \\
\hline 5 & Molecular area $\left(\AA^{2}\right)$ & 285.727 & 285.723 & 284.149 \\
\hline 6 & Ionisation potential $(I)$ & 5.9866 & 6.0683 & 6.0139 \\
\hline 7 & Electron affinity $(A)$ & 1.8504 & 1.8776 & 1.7960 \\
\hline 8 & Hardness $(\eta)$ & 2.0681 & 2.0953 & 2.1089 \\
\hline 9 & Softness $(\sigma)$ & 0.4835 & 0.4773 & 0.4742 \\
\hline 10 & Electronegativity $(\chi)$ & 3.9185 & 3.9730 & 3.9049 \\
\hline 11 & Electrophilicity index $(\omega)$ & 3.7123 & 3.7666 & 3.6152 \\
\hline 12 & Fractions of electron transferred $(\Delta N)$ & 1.0172 & 0.9910 & 1.0008 \\
\hline 13 & Dipole moment $\mu(\mathrm{D})$ & 4.3221 & 3.8206 & 2.6046 \\
\hline 14 & $\Delta E_{\text {backdonation }}(\mathrm{eV})$ & -0.517 & -0.5238 & -0.5272 \\
\hline
\end{tabular}

highest fraction of electrons transferred is associated with the best inhibitor (4-CNMBA). Ionization energy is a fundamental descriptor of the chemical reactivity of atoms and molecules. High ionization energy indicates high stability and chemical inertness, while small ionization energy indicates high reactivity of the atoms and molecules. The low ionization energy of 4-CNMBA indicates its high inhibition efficiency.

The dipole moment $(\mu)$ provides information about the polarity of the whole molecule. High dipole moment means greater molecular polarity, which probably gives rise to high chemical reactivity [32]. It relates to the dipole-dipole interaction of inhibitor molecule and metal surface. Table 2 shows that 4-CNMBA has the highest dipole moment. it is due to electron releasing effect ( $+\mathrm{R}$ effect) of $-\mathrm{OCH}_{3}$ and electron withdrawing effect of $-\mathrm{Cl}$ atom ( $-\mathrm{I}$ effect), both of which are at the para position to the benzene rings attached to the $>\mathrm{C}=\mathrm{N}$ group which will increase its molecular reactivity, leading to stronger adsorption onto the metal surface compared to the adsorption of 3-CNMBA and 2-CNMBA.

The unoccupied orbitals of zinc can accept electrons from inhibitor molecules to form a coordinate bond. Also the inhibitor molecules can accept electrons from zinc atom using its antibonding orbitals to form backdonating bond. These donation and backdonation processes strengthen the adsorption of these inhibitors onto the zinc surface. When $\eta>0$ and $\Delta E_{\text {backdonation }}<0$, the charge transferred to a molecule followed by backdonation from a molecule is energetically favourable. In Table 2 , the calculated $\Delta E_{\text {backdonation }}$ values for the inhibitors followed the order 4-CNMBA > 3$\mathrm{CNMBA}>2$-CNMBA which indicates that backdonation is favoured for the 4-CNMBA, which is the best inhibitor.

As we know, frontier orbital theory is useful in predicting the adsorption centres of the inhibitors responsible for the interaction with surface metal atoms. Figures 4(a), 4(b), and 4(c) show the HOMO and LUMO orbital contributions for the neutral species of the studied inhibitor molecules 4CNMBA, 3-CNMBA, and 2-CNMBA, respectively. For all the molecules, the HOMO densities were concentrated on both rings, $\mathrm{Cl}$ atom, $>\mathrm{C}=\mathrm{N}$ group, and $\mathrm{O}$-atom of methoxy group. This means that these are active sites of the molecules responsible for interaction with metal surface.

The LUMO molecular orbital was uniformly distributed over rings as well as on $>\mathrm{C}=\mathrm{N}$ group, while contribution from $\mathrm{Cl}$ atom is negligible. Due to the presence of an iminic $(>\mathrm{C}=\mathrm{N}-$ ) group and rings, these molecules could function as good inhibitors.

Local charges are important in many chemical reactions and physicochemical properties of compound [26]. Tables 3, 4 , and 5 show that most of the carbon atoms $(\mathrm{C} 1, \mathrm{C} 2, \mathrm{C} 3$, $\mathrm{C} 4, \mathrm{C} 5, \mathrm{C} 12, \mathrm{C} 20, \mathrm{C} 21, \mathrm{C} 22, \mathrm{C} 23$, and C25), oxygen atom (O11), nitrogen atom (N18), and chlorine atom (Cl29 in case of 3-CNMBA and 4-CNMBA) possess the excess of negative charge. Among them, highest negative charge is located on the nitrogen (N18) and oxygen atom (O11), in all the three studied inhibitors. It confirmed that electron donation ability of atoms to the unoccupied orbital of metal is directly related to negative atomic charges of adsorption sites. Therefore, these atoms should be the active adsorption sites.

The preferred site for nucleophilic attack is the atom in the molecule where Fukui function $\left(f^{+}\right)$has the highest value as it is associated with the LUMO and measures the reactivity towards a donor reagent. The preferred site for electrophilic attack is the atom in the molecule where the value of Fukui function $\left(f^{-}\right)$is the highest as it is associated with the $\mathrm{HOMO}$ and measures reactivity toward an acceptor reagent. For radical attack, the preferred site is one with highest $f^{o}$ value. An analysis of Fukui functions depicted in Tables 3-5 shows that the atoms O11 and N18 are preferred sites for electrophilic attack, as these sites have higher values of Fukui function $\left(f^{-}\right)$. The atoms C6, C16, and O11 are most susceptible sites for nucleophilic attack since these sites have highest values of Fukui function $\left(f^{+}\right)$. Chlorine $\mathrm{Cl} 29$ has very low values of Fukui indices. For the simplest transfer of electrons, adsorption could occur at the part of the molecule, where local softness $(s)$ has the highest value. A high value of $s^{+}$indicates high nucleophilicity and a high value of $s^{-}$ 

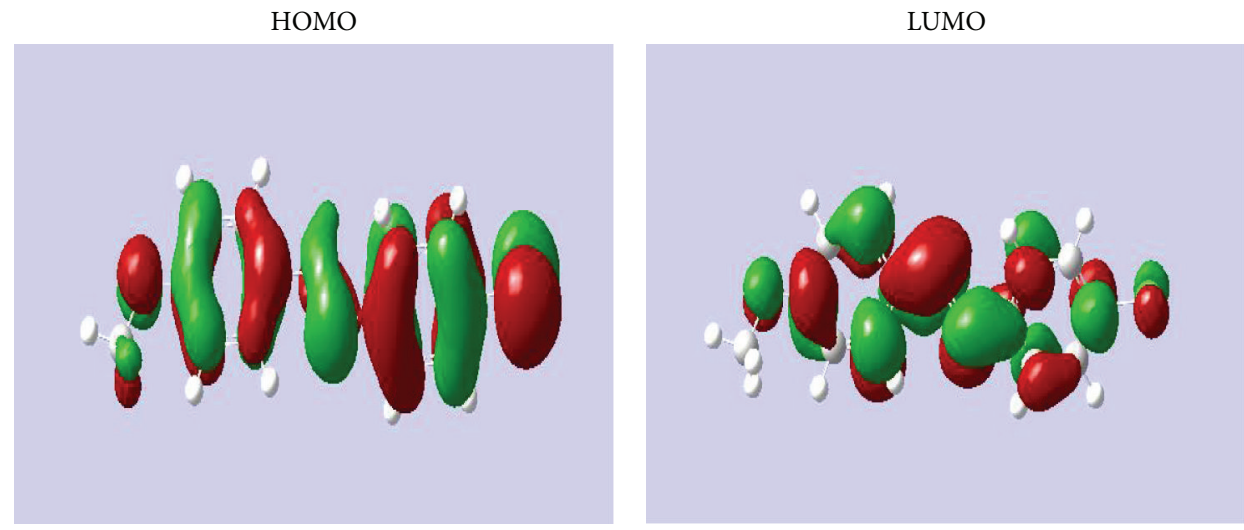

(a) 4-CNMBA
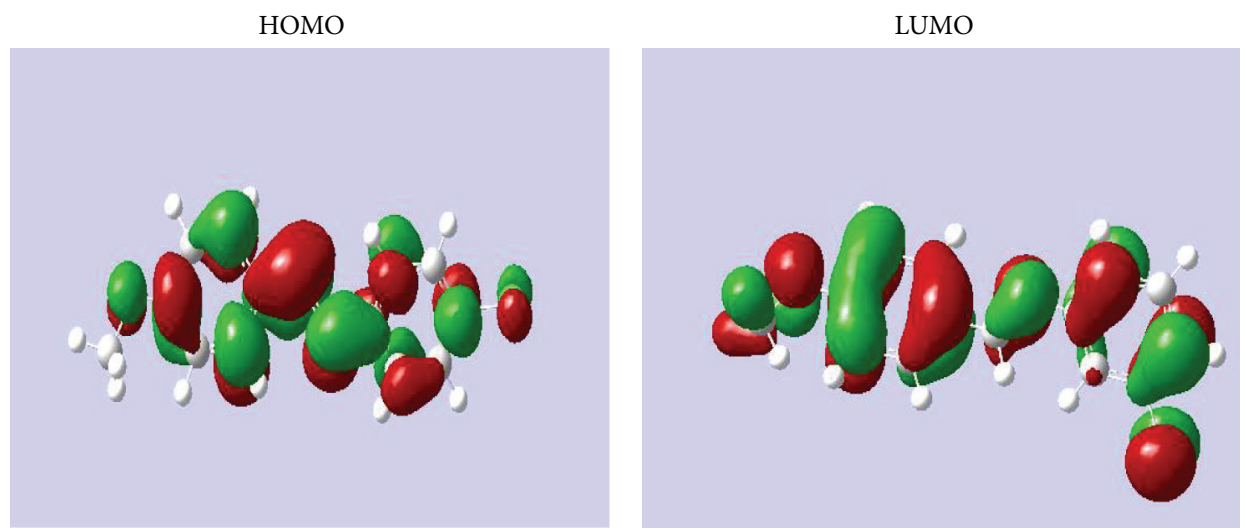

(b) 3-CNMBA

HOMO

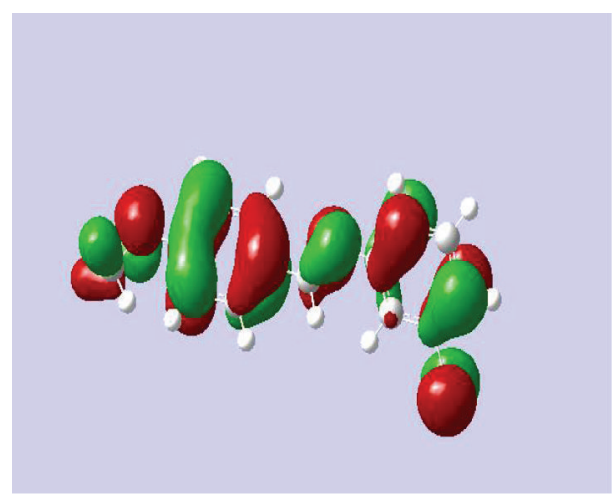

LUMO

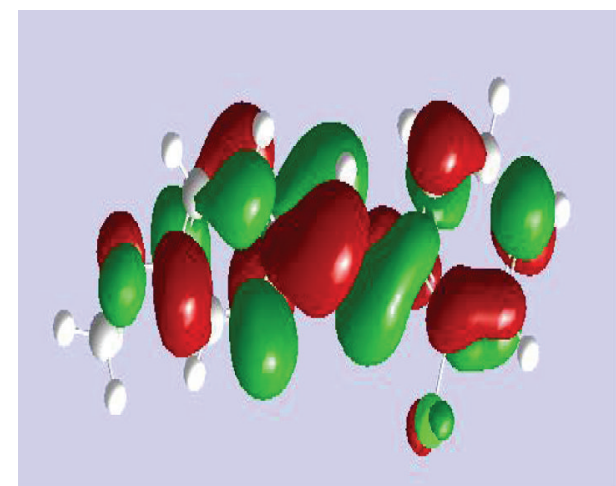

(c) 2-CNMBA

FIGURE 4: HOMO and LUMO diagrams of studied compounds using B3LYP/6-31+G(d) method.

indicates high electrophilicity. The results shown in Tables 35 indicate that the condensed local softness indices $s^{+}$and $s^{-}$ follow the same trend as that of Fukui functions.

\subsection{Quantitative Structure and Activity Relationship (QSAR)} Consideration. Theoretical results discussed above show that there is no simple relation between quantum chemical parameters and inhibition efficiency of studied inhibitors. In order to correlate the quantum chemical parameters of the inhibitors and their experimental inhibition efficiencies, attempts were made to fit \% IE to linear and nonlinear models by using XLSTAT $[19,33]$. The following quantum chemical parameters were considered; these were the energy of the highest occupied molecular orbital $E_{\mathrm{HOMO}}(\mathrm{eV})$, energy of lowest unoccupied molecular orbital $E_{\mathrm{LUMO}}(\mathrm{eV})$, energy gap $\Delta E$ $(\mathrm{eV})$, and the dipole moment $\mu$ (D). The nonlinear regression 
TABLE 3: Calculated natural charges, Fukui functions, and local softness indices for the schiff base (4-CNMBA).

\begin{tabular}{|c|c|c|c|c|c|c|c|c|c|}
\hline \multicolumn{2}{|c|}{ Atom } & $q_{N}$ & $q_{N+1}$ & $q_{N-1}$ & $f^{+}$ & $f^{-}$ & $f^{o}$ & $s^{+}$ & $s^{-}$ \\
\hline 1 & $\mathrm{C}$ & -0.2196 & -0.14569 & -0.04264 & 0.07391 & -0.17696 & -0.05153 & 0.035735 & -0.08556 \\
\hline 2 & $\mathrm{C}$ & -0.24488 & -0.13166 & -0.13084 & 0.11322 & -0.11404 & -0.00041 & 0.054742 & -0.05514 \\
\hline 3 & $\mathrm{C}$ & -0.06522 & -0.12044 & 0.08584 & -0.05522 & -0.15106 & -0.10314 & -0.0267 & -0.07304 \\
\hline 4 & $\mathrm{C}$ & -0.24402 & -0.11844 & -0.11751 & 0.12558 & -0.12651 & -0.00047 & 0.060718 & -0.06117 \\
\hline 5 & $\mathrm{C}$ & -0.24886 & -0.17656 & -0.06871 & 0.0723 & -0.18015 & -0.05393 & 0.034957 & -0.0871 \\
\hline 6 & $\mathrm{C}$ & 0.12038 & 0.03884 & 0.11527 & -0.08154 & 0.00511 & -0.03822 & -0.03942 & 0.002471 \\
\hline 7 & $\mathrm{~N}$ & -0.45311 & -0.35283 & -0.09553 & 0.10028 & -0.35758 & -0.12865 & 0.048485 & -0.17289 \\
\hline 8 & $\mathrm{C}$ & 0.11904 & -0.15754 & 0.05276 & -0.27658 & 0.06628 & -0.10515 & -0.13373 & 0.032046 \\
\hline 9 & $\mathrm{C}$ & -0.15354 & -0.07595 & 0.01912 & 0.07759 & -0.17266 & -0.04754 & 0.037515 & -0.08348 \\
\hline 10 & $\mathrm{C}$ & -0.1888 & -0.14648 & -0.09176 & 0.04232 & -0.09704 & -0.02736 & 0.020462 & -0.04692 \\
\hline 11 & $\mathrm{C}$ & -0.27749 & -0.13428 & -0.09148 & 0.14321 & -0.18601 & -0.0214 & 0.069242 & -0.08994 \\
\hline 12 & $\mathrm{C}$ & 0.33473 & 0.03742 & 0.2429 & -0.29731 & 0.09183 & -0.10274 & -0.14375 & 0.0444 \\
\hline 13 & $\mathrm{C}$ & -0.3188 & -0.12951 & -0.1354 & 0.18929 & -0.1834 & 0.002945 & 0.091522 & -0.08867 \\
\hline 14 & $\mathrm{C}$ & -0.16776 & -0.21797 & -0.05563 & -0.05021 & -0.11213 & -0.08117 & -0.02428 & -0.05421 \\
\hline 15 & $\mathrm{Cl}$ & -0.00453 & -0.03752 & 0.09301 & -0.03299 & -0.09754 & -0.06527 & -0.01595 & -0.04716 \\
\hline 16 & $\mathrm{O}$ & -0.5322 & -0.29037 & -0.17715 & 0.24183 & -0.35505 & -0.05661 & 0.116925 & -0.17167 \\
\hline 17 & $\mathrm{C}$ & -0.32732 & -0.15824 & -0.17362 & 0.16908 & -0.1537 & 0.00769 & 0.08175 & -0.07431 \\
\hline
\end{tabular}

TABLE 4: Calculated natural charges, Fukui functions, and local softness indices for the schiff base (3-CNMBA).

\begin{tabular}{|c|c|c|c|c|c|c|c|c|c|}
\hline \multicolumn{2}{|c|}{ Atom } & \multirow{2}{*}{$\frac{q_{N}}{-0.23802}$} & \multirow{2}{*}{$\frac{q_{N+1}}{-0.15223}$} & \multirow{2}{*}{$\frac{q_{N-1}}{-0.00325}$} & \multirow{2}{*}{$\frac{f^{+}}{0.08579}$} & \multirow{2}{*}{$\begin{array}{c}f^{-} \\
-0.23477\end{array}$} & \multirow{2}{*}{$\begin{array}{c}f^{o} \\
-0.07449\end{array}$} & \multirow{2}{*}{$\frac{s^{+}}{0.040948}$} & \multirow{2}{*}{$\frac{s^{-}}{-0.11206}$} \\
\hline 1 & $\mathrm{C}$ & & & & & & & & \\
\hline 2 & $\mathrm{C}$ & -0.22184 & -0.12141 & -0.14476 & 0.10043 & -0.07708 & 0.011675 & 0.047935 & -0.03679 \\
\hline 3 & $\mathrm{C}$ & -0.26326 & -0.22868 & 0.02666 & 0.03458 & -0.28992 & -0.12767 & 0.016505 & -0.13838 \\
\hline 4 & $\mathrm{C}$ & -0.04642 & -0.01673 & -0.02292 & 0.02969 & -0.0235 & 0.003095 & 0.014171 & -0.01122 \\
\hline 5 & $\mathrm{C}$ & -0.27494 & -0.18587 & -0.10333 & 0.08907 & -0.17161 & -0.04127 & 0.042513 & -0.08191 \\
\hline 6 & $\mathrm{C}$ & 0.13715 & 0.04358 & 0.10267 & -0.09357 & 0.03448 & -0.02955 & -0.04466 & 0.016457 \\
\hline 7 & $\mathrm{~N}$ & -0.45246 & -0.3519 & -0.08289 & 0.10056 & -0.36957 & -0.13451 & 0.047997 & -0.1764 \\
\hline 8 & $\mathrm{C}$ & 0.12165 & -0.15952 & 0.04464 & -0.28117 & 0.07701 & -0.10208 & -0.1342 & 0.036757 \\
\hline 9 & $\mathrm{C}$ & -0.15444 & -0.14618 & 0.03144 & 0.00826 & -0.18588 & -0.08881 & 0.003942 & -0.08872 \\
\hline 10 & $\mathrm{C}$ & -0.18766 & -0.22069 & -0.09419 & -0.03303 & -0.09347 & -0.06325 & -0.01577 & -0.04461 \\
\hline 11 & $\mathrm{C}$ & -0.27739 & -0.30396 & -0.08569 & -0.02657 & -0.1917 & -0.10914 & -0.01268 & -0.0915 \\
\hline 12 & $\mathrm{C}$ & 0.33564 & 0.03726 & 0.2462 & -0.29838 & 0.08944 & -0.10447 & -0.14242 & 0.04269 \\
\hline 13 & $\mathrm{C}$ & -0.3189 & -0.12939 & -0.13187 & 0.18951 & -0.18703 & 0.00124 & 0.090453 & -0.08927 \\
\hline 14 & $\mathrm{C}$ & -0.16701 & -0.21838 & -0.05691 & -0.05137 & -0.1101 & -0.08074 & -0.02452 & -0.05255 \\
\hline 15 & $\mathrm{Cl}$ & -0.00679 & -0.03136 & 0.04529 & -0.02457 & -0.05208 & -0.03833 & -0.01173 & -0.02486 \\
\hline 16 & $\mathrm{O}$ & -0.53188 & -0.29041 & -0.16938 & 0.24147 & -0.3625 & -0.06052 & 0.115254 & -0.17302 \\
\hline 17 & $\mathrm{C}$ & -0.32737 & -0.15822 & -0.17437 & 0.16915 & -0.153 & 0.008075 & 0.080735 & -0.07303 \\
\hline
\end{tabular}

using polynomial equation of degree two yielded nearly as good results as found by experiments. The polynomial model yielded the following

$$
\begin{gathered}
\% \mathrm{IE}_{\mathrm{pred}}=-37.3+\left(-48875.9 E_{\mathrm{HOMO}}+45332.8 E_{\mathrm{LUMO}}\right. \\
-50332.6 \Delta E+164.4 \mu) C_{\mathrm{inh}} \\
+\left(403.5 E_{\mathrm{HOMO}}^{2}-4605.9 E_{\mathrm{LUMO}}^{2}-90.9 \mu^{2}\right) C_{\mathrm{inh}}^{2}, \\
R^{2}=0.996, \quad \mathrm{RMSE}=1.27,
\end{gathered}
$$

where $C_{\text {inh }}$ is the concentration of inhibitor, $R^{2}$ is the coefficient of determination, and RMSE is defined as

$$
\mathrm{RMSE}=\sqrt{\frac{1}{n} \sum_{i=1}^{n}\left(\mathrm{IE}_{\text {pred }}-\mathrm{IE}_{\mathrm{exp}}\right)^{2}},
$$

where $I E_{\text {pred }}$ is the predicted inhibition efficiency, $I_{\text {exp }}$ is the experimental inhibition efficiency, and $n$ is the number of observations considered.

The experimental and calculated efficiencies are listed in Figure 5. The present model was successfully used to predict corrosion inhibition efficiencies of zinc in acidic medium for intermediate concentrations of inhibitors. It was clearly 
TABLE 5: Calculated natural charges, Fukui functions, and local softness indices for the schiff base (2-CNMBA).

\begin{tabular}{|c|c|c|c|c|c|c|c|c|c|}
\hline \multicolumn{2}{|c|}{ Atom } & $q_{N}$ & $q_{N+1}$ & $q_{N-1}$ & $f^{+}$ & $f^{-}$ & $f^{o}$ & $s^{+}$ & $s^{-}$ \\
\hline 1 & $\mathrm{C}$ & -0.25079 & -0.16365 & -0.08941 & 0.08714 & -0.16138 & 0.03712 & 0.041322 & -0.07653 \\
\hline 2 & $\mathrm{C}$ & -0.23737 & -0.12167 & -0.10027 & 0.1157 & -0.1371 & 0.0107 & 0.054865 & -0.06501 \\
\hline 3 & $\mathrm{C}$ & -0.24278 & -0.21169 & 0.0102 & 0.03109 & -0.25298 & 0.110945 & 0.014743 & -0.11996 \\
\hline 4 & $\mathrm{C}$ & -0.24284 & -0.12075 & -0.1506 & 0.12209 & -0.09224 & -0.01493 & 0.057895 & -0.04374 \\
\hline 5 & $\mathrm{C}$ & -0.0593 & -0.06755 & 0.05873 & -0.00825 & -0.11803 & 0.06314 & -0.00391 & -0.05597 \\
\hline 6 & $\mathrm{C}$ & 0.11317 & 0.03661 & 0.10637 & -0.07656 & 0.0068 & 0.03488 & -0.0363 & 0.003225 \\
\hline 7 & $\mathrm{~N}$ & -0.44314 & -0.35471 & -0.07596 & 0.08843 & -0.36718 & 0.139375 & 0.041934 & -0.17412 \\
\hline 8 & $\mathrm{C}$ & 0.12386 & -0.15875 & 0.04714 & -0.28261 & 0.07672 & 0.102945 & -0.13401 & 0.036381 \\
\hline 9 & $\mathrm{Cl}$ & 0.01263 & -0.01662 & 0.07567 & -0.02925 & -0.06304 & 0.046145 & -0.01387 & -0.02989 \\
\hline 10 & $\mathrm{C}$ & -0.155 & -0.07767 & 0.02588 & 0.07733 & -0.18088 & 0.051775 & 0.03667 & -0.08577 \\
\hline 11 & $\mathrm{C}$ & -0.18812 & -0.14794 & -0.09464 & 0.04018 & -0.09348 & 0.02665 & 0.019053 & -0.04433 \\
\hline 12 & $\mathrm{C}$ & -0.27813 & -0.13424 & -0.08709 & 0.14389 & -0.19104 & 0.023575 & 0.068233 & -0.09059 \\
\hline 13 & $\mathrm{C}$ & 0.33556 & 0.03347 & 0.24292 & -0.30209 & 0.09264 & 0.104725 & -0.14325 & 0.04393 \\
\hline 14 & $\mathrm{C}$ & -0.31875 & -0.12844 & -0.13326 & 0.19031 & -0.18549 & -0.00241 & 0.090245 & -0.08796 \\
\hline 15 & $\mathrm{C}$ & -0.16547 & -0.21981 & -0.05762 & -0.05434 & -0.10785 & 0.081095 & -0.02577 & -0.05114 \\
\hline 16 & $\mathrm{O}$ & -0.53224 & -0.29096 & -0.17334 & 0.24128 & -0.3589 & 0.05881 & 0.114415 & -0.17019 \\
\hline 17 & $\mathrm{C}$ & -0.32738 & -0.15817 & -0.17402 & 0.16921 & -0.15336 & -0.00793 & 0.080239 & -0.07272 \\
\hline
\end{tabular}

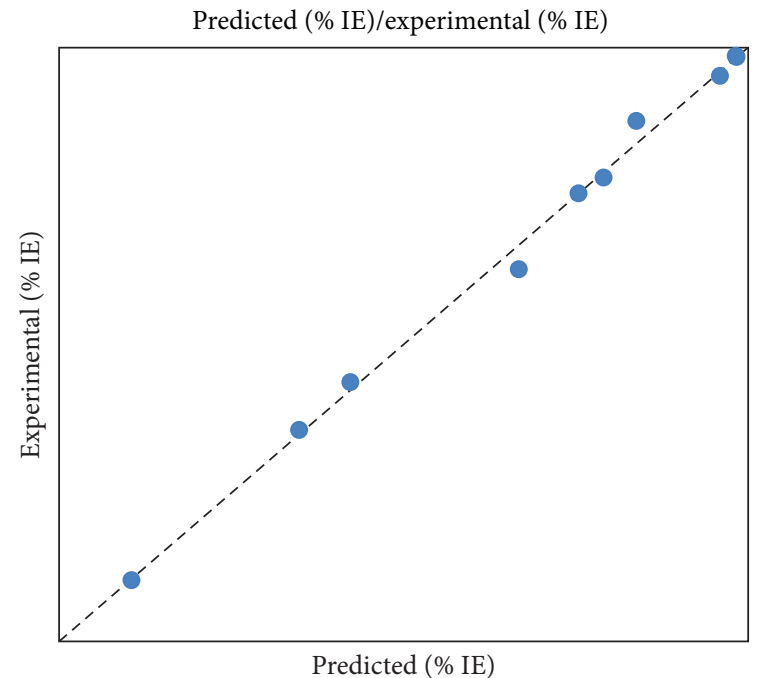

FIGURE 5: Experimental and predicted (\% IE) of studied inhibitors by using regression (11).

insufficient at very high concentrations where efficiencies do not increase with increase in concentrations of the inhibitors.

Inhibition efficiency is best described by dipole moment $(\mu)$ and remaining three variables $E_{\mathrm{HOMO}}, E_{\mathrm{LUMO}}$, and $\Delta E$ equally contribute in predicting inhibition efficiency.

\section{Conclusions}

4-CNMBA, 3-CNMBA, and 2-CNMBA are effective inhibitors of corrosion of zinc exposed to $0.5 \mathrm{M} \mathrm{HCl}$ solution. Their inhibition efficiencies increase in the order of 4-CNMBA > 3-CNMBA > 2-CNMBA. The inhibition efficiencies tend to increase by increasing the inhibitor concentration. Percent inhibition efficiencies were explained in terms of molecular parameters. Since the calculated quantum chemical properties can change by an addition of a functional group to the molecule, the correlations between inhibition efficiency and molecular parameters can be used for preselection of new inhibitors. Understanding the phenomena of adsorption is key point in corrosion problems. Computational studies help to find the most probable adsorption sites for a molecule. This information can help to gain further insight into corrosion system, such as the most probable site for inhibitor adsorption. A QSAR equation was developed and used to predict the corrosion inhibition efficiency for studied inhibitors. The experimental measurements match the prediction of corrosion efficiencies of these compounds. QSAR approach may be useful to predict and design the structure and molecule that is suitable to be an effective corrosion inhibitor.

\section{Conflict of Interests}

The authors declare that there is no conflict of interests regarding the publication of this paper.

\section{Acknowledgments}

The authors are grateful to the Chemistry Department, School of Sciences, Gujarat University, Gujarat, for the laboratory facilities and to the Central University of Gujarat for providing computational support.

\section{References}

[1] I. B. Obot, N. O. Obi-Egbedi, and S. A. Umoren, "Adsorption characteristics and corrosion inhibitive properties of clotrimazole for aluminium corrosion in hydrochloric acid," International Journal of Electrochemical Science, vol. 4, no. 6, pp. 863877, 2009. 
[2] S. G. Zhang, W. Lei, M. Z. Xia, and F. Y. Wang, "QSAR study on N-containing corrosion inhibitors: quantum chemical approach assisted by topological index," Journal of Molecular Structure: THEOCHEM, vol. 732, no. 1-3, pp. 173-182, 2005.

[3] K. F. Khaled, "Adsorption and inhibitive properties of a new synthesized guanidine derivative on corrosion of copper in $0.5 \mathrm{M} \mathrm{H}_{2} \mathrm{SO}_{4}$," Applied Surface Science, vol. 255, no. 5, pp. 18111818, 2008.

[4] R. T. Vashi, H. M. Bhajiwala, and S. A. Desai, "Ethanolamines as corrosion inhibitors for Zinc in $\left(\mathrm{HNO}_{3}+\mathrm{H}_{2} \mathrm{SO}_{4}\right)$ binary acid mixture," E-Journal of Chemistry, vol. 7, no. 2, pp. 665-668, 2010.

[5] K. K. Taha and A. Muhideen, "Characterization of anticorrosion triazole film," The Journal of Science \& Technology, vol. 10, pp. 92-99, 2009.

[6] G. W. Walter, "Corrosion rates of zinc, zinc coatings and steel in aerated slightly acidic chloride solutions calculated from low polarization data," Corrosion Science, vol. 16, no. 9, pp. 573-586, 1976.

[7] L. Wang, J.-X. Pu, and H.-C. Luo, "Corrosion inhibition of zinc in phosphoric acid solution by 2 -mercaptobenzimidazole," Corrosion Science, vol. 45, no. 4, pp. 677-683, 2003.

[8] S. Liu, Y. Zhong, R. Jiang, Z. Zeng, Z. Feng, and R. Xiao, “Corrosion inhibition of zinc in tetra-n-butylammonium bromide aerated aqueous solution by benzotriazole and $\mathrm{Na}_{3} \mathrm{PO}_{4}$," Corrosion Science, vol. 53, no. 2, pp. 746-759, 2011.

[9] E. C. Ogoko, S. A. Odoemelam, B. I. Ita, and N. O. Eddy, "Adsorption and inhibitive properties of clarithromycin for the corrosion of $\mathrm{Zn}$ in 0.01 to $0.05 \mathrm{M} \mathrm{H}_{2} \mathrm{SO}_{4}$," Portugaliae Electrochimica Acta, vol. 27, no. 6, pp. 713-724, 2009.

[10] Y. K. Agrawal, J. D. Talati, M. D. Shah, M. N. Desai, and N. K. Shah, "Schiff bases of ethylenediamine as corrosion inhibitors of zinc in sulphuric acid," Corrosion Science, vol. 46, no. 3, pp. 633-651, 2004.

[11] M. S. Abdel-All, Z. A. Ahmed, and M. S. Hassan, "Inhibiting and accelerating effects of some quinolines on the corrosion of zinc and some binary zinc alloys in $\mathrm{HCl}$ solution," Journal of Applied Electrochemistry, vol. 22, no. 11, pp. 1104-1109, 1992.

[12] O. K. Abiola and A. O. James, "The effects of Aloe vera extract on corrosion and kinetics of corrosion process of zinc in $\mathrm{HCl}$ solution," Corrosion Science, vol. 52, no. 2, pp. 661-664, 2010.

[13] M. Abdallah, A. Y. El-Etre, and M. F. Moustafa, "Amidopoly ethylamines as corrosion inhibitors for zinc dissolution in different acidic electrolytes," Portugaliae Electrochimica Acta, vol. 27, no. 5, pp. 615-630, 2009.

[14] M. D. Shah, A. S. Patel, G. V. Mudaliar, and N. K. Shah, "Schiff bases of triethylenetetramine as corrosion inhibitors of Zinc in Hydrochloric acid," Portugaliae Electrochimica Acta, vol. 29, no. 2, pp. 101-113, 2011.

[15] A. Y. Musa, R. T. T. Jalgham, and A. B. Mohamad, "Molecular dynamic and quantum chemical calculations for phthalazine derivatives as corrosion inhibitors of mild steel in $1 \mathrm{M} \mathrm{HCl}$," Corrosion Science, vol. 56, pp. 176-183, 2012.

[16] M. J. Frisch, G. W. Trucks, H. B. Schlegel et al., Gaussian 09, Revision A.1, Gaussian, Inc., Wallingford, Conn, USA, 2009.

[17] A. Rauk, Orbital Interaction Theory of Organic Chemistry, John Wiley \& Sons, New York, NY, USA, 2nd edition, 2001.

[18] T. A. Carlson, C. W. Nestor Jr., N. Wasserman, and J. D. Mcdowell, "Calculated ionization potentials for multiply charged ions," Atomic Data and Nuclear Data Tables, vol. 2, pp. 63-99, 1970.

[19] M. M. Kabanda, L. C. Murulana, M. Ozcan et al., "Quantum chemical studies on the corrosion inhibition of mild steel by some triazoles and benzimidazole derivatives in acidic medium," International Journal of Electrochemical Science, vol. 7, pp. 5035-5056, 2012.

[20] R. G. Pearson, "Absolute electronegativity and hardness: application to inorganic chemistry," Inorganic Chemistry, vol. 27, no. 4, pp. 734-740, 1988.

[21] R. G. Pearson, "Chemical hardness and bond dissociation energies," Journal of the American Chemical Society, vol. 110, no. 23, pp. 7684-7690, 1988.

[22] Z. E. Adnani, M. Mcharfi, M. Sfaira, M. Benzakour, A. T. Benjelloun, and M. E. Touhami, "DFT theoretical study of 7-R3methylquinoxalin-2 $(1 \mathrm{H})$-thiones $\left(\mathrm{R}=\mathrm{H} ; \mathrm{CH}_{3} ; \mathrm{Cl}\right)$ as corrosion inhibitors in hydrochloric acid," Corrosion Science, vol. 68, pp. 223-230, 2013.

[23] I. B. Obot and N. O. Obi-Egbedi, "Theoretical study of benzimidazole and its derivatives and their potential activity as corrosion inhibitors," Corrosion Science, vol. 52, no. 2, pp. 657660, 2010.

[24] P. Udhayakala, T. V. Rajendiran, and S. Gunasekaran, "Theoretical evaluation on the efficiencies of some. Flavonoids as corrosion inhibitors on copper," Journal of Chemical, Biological and Physical Sciences A, vol. 2, no. 3, pp. 1151-1165, 2012.

[25] R. G. Parr, L. V. Szentpály, and S. Liu, "Electrophilicity index," Journal of the American Chemical Society, vol. 121, no. 9, pp. 1922-1924, 1999.

[26] K. F. Khaled, "Experimental, density function theory calculations and molecular dynamics simulations to investigate the adsorption of some thiourea derivatives on iron surface in nitric acid solutions," Applied Surface Science, vol. 256, no. 22, pp. 6753-6763, 2010.

[27] E. E. Ebenso, D. A. Isabirye, and N. O. Eddy, "Adsorption and quantum chemical studies on the inhibition potentials of some thiosemicarbazides for the corrosion of mild steel in acidic medium," International Journal of Molecular Sciences, vol. 11, no. 6, pp. 2473-2498, 2010.

[28] B. Gomez, N. V. Likhanova, M. A. Dominguez-Anuilar, R. Martínez-Palou, A. Vela, and J. L. Gázquez, "Quantum chemical study of the inhibitive properties of 2-pyridyl-azoles," The Journal of Physical Chemistry B, vol. 110, no. 18, pp. 8928-8934, 2006.

[29] P. Udhayakala, T. V. Rajendiran, and S. Gunasekaran, “Theoretical evaluation of corrosion inhibition performance of some triazole derivatives," Journal of Advanced Scientific Research, vol. 3, pp. 71-77, 2012.

[30] N. O. Obi-Egbedi, I. B. Obot, M. I. El-Khaiary, S. A. Umoren, and E. E. Ebenso, "Computational simulation and statistical analysis on the relationship between corrosion inhibition efficiency and molecular structure of some phenanthroline derivatives on mild steel surface," International Journal of Electrochemical Science, vol. 6, no. 11, pp. 5649-5675, 2011.

[31] M. Özcan, R. Solmaz, G. Kardaş, and I. Dehri, "Adsorption properties of barbiturates as green corrosion inhibitors on mild steel in phosphoric acid," Colloids and Surfaces A, vol. 325, no. 1-2, pp. 57-63, 2008.

[32] O. Kikuchi, "Systematic QSAR procedures with quantum chemical descriptors," Quantitative Structure-Activity Relationships, vol. 6, no. 4, pp. 179-184, 1987.

[33] I. Lukovits, I. Bakó, A. Shaban, and E. Kálmán, "Polynomial model of the inhibition mechanism of thiourea derivatives," Electrochimica Acta, vol. 43, no. 1-2, pp. 131-136, 1998. 

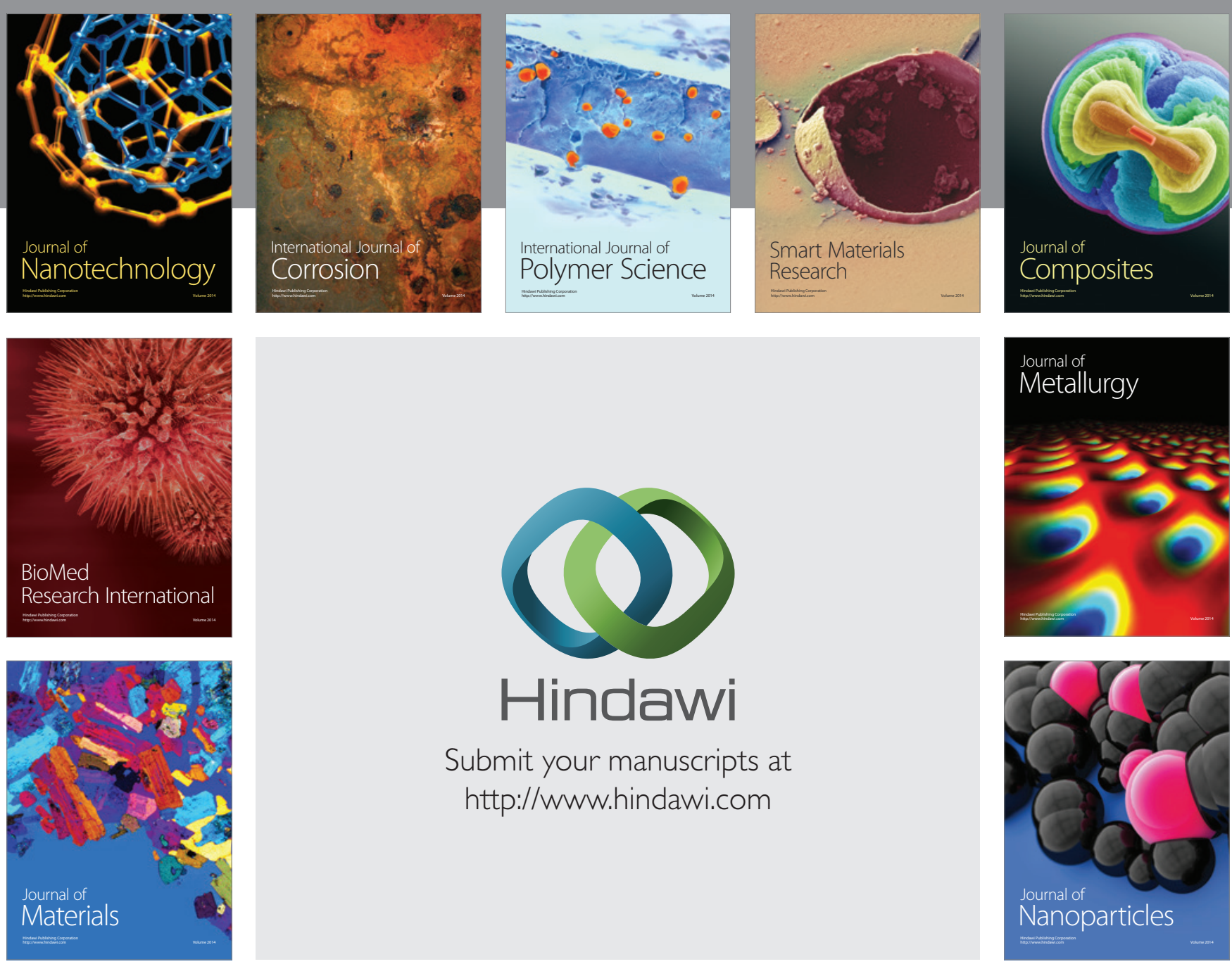

Submit your manuscripts at http://www.hindawi.com
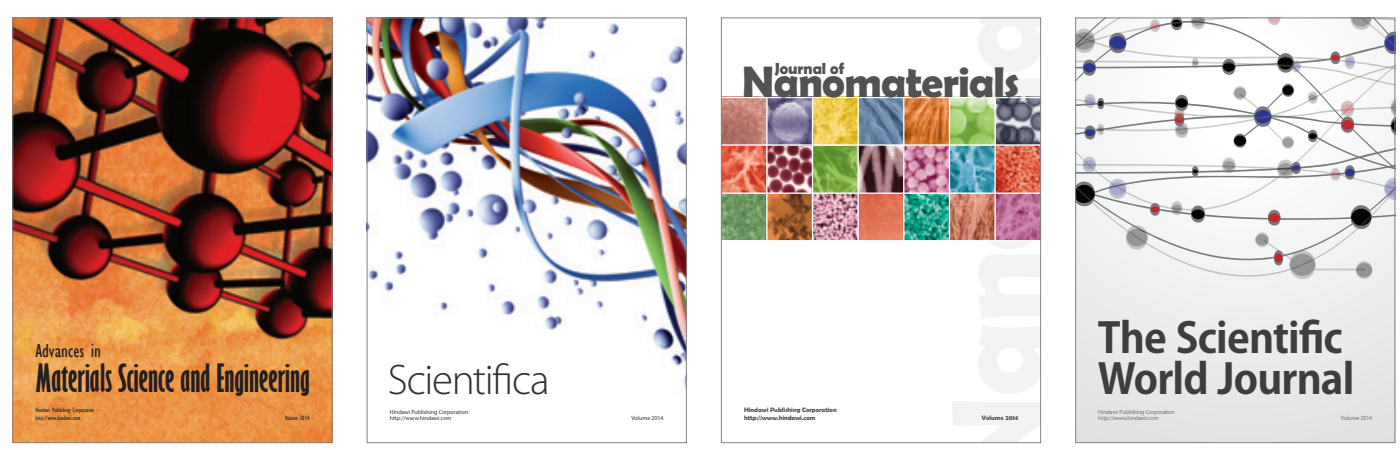

\section{The Scientific World Journal}
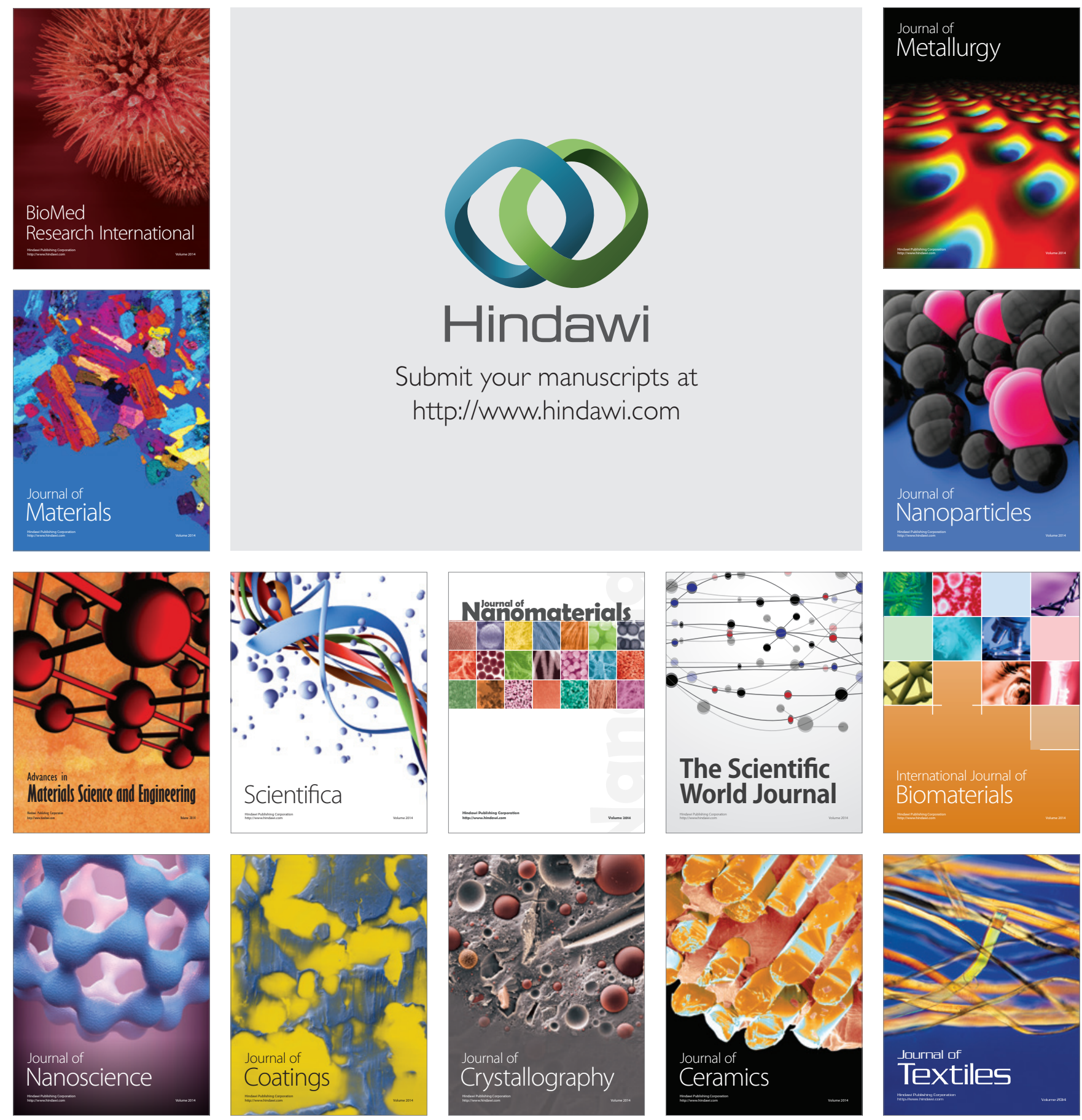